\title{
Simple Models of Foundation-Soil Interactions
}

\author{
Shi-Shuenn Chen and Jun-Yang Shi
}

\begin{abstract}
This study aims to develop a series of simplified mass-spring-dashpot model to simulate unbounded soil for a foundation-soil system subjected to vertical, horizontal, rocking and torsional motions. A group of equivalent models are established by using three equivalent criteria and the coupling of horizontal and rocking motions is also considered. An optimal equivalent model is then determined to represent the best simplified model. The dynamic responses of the foundation-soil system using the optimal equivalent model are compared with those obtained by the half-space theory and by the lumped-parameter models. Since the coupling of horizontal and rocking motions is adequately considered, the optimal equivalent model is found to have more accurate results than most existing models. Moreover, the proposed model is also applied to a time-history analysis for a building-soil interaction system subjected to horizontal excitations. This proposed method may be effectively applicable to practical soil-structure interaction problems.
\end{abstract}

Index Terms-Foundation vibration, lumped-parameter model, soil-structure interaction, seismic analysis

\section{INTRODUCTION}

The most commonly-used methods for dynamic soil-structure interaction (SSI) analysis are the complete method and the substructure method. The complete method features in analyzing the response of soil and structures simultaneously, which usually requires special boundaries to simulate the unbounded soil. In contrast, the substructure method features in analyzing soil and structures separately, which generally uses dynamic impedance functions to represent the soil behavior. The dynamic impedance function is the force-displacement relationship for interaction points between foundation and soil. However, since the dynamic impedances are frequency-dependent, they can not be used directly in time-domain structural analysis. Hence, simplified models with frequency-independent parameters are extensively developed for effectively simulating the unbounded soil in time domain.

One type of the simplified models is a lumped-parameter model, which comprises masses, springs, and dashpots. Through a curve-fitting technique or an optimization process, the modeling parameters of the lumped-parameter model are obtained by minimizing the discrepancy between the impedance functions for the model and that obtained from the rigorous theory. Then a lumped-parameter model is developed for a foundation-soil system while the foundation

Manuscript received April 10, 2013; revised May 29, 2013. This work was supported in part by the National Science Council of the Republic of China under Grant No. NSC 101-2221-E-011-082.

The authors are with the Department of Construction Engineering, National Taiwan University of Science and Technology, Taiwan (e-mail: sschen@mail.ntust.edu.tw, D9105001@mail.ntust.edu.tw). is massless. Several studies [1]-[11] followed the conventional method addressed above to develop the simplified models. The conventional method used an optimization process to determine the unknown parameters of the lumped-parameter models so that the accuracies of those models were affected by objective functions used in optimization. Since the dynamic impedances in low-to-medium frequencies are more important on the system response, Wolf [7] suggested that the objective functions need to be adapted to make the weight for the low frequencies higher than that for the high frequencies. However, the relation between the frequency and the weight was not clearly clarified. Barros and Luco [2] suggested that an iteration method be adopted to determine an appropriate weight in the objective function until the impedance function of the simplified model approaches theoretical solutions well. This approach is relatively time-consuming.

This paper aims to presents a new simplified model to simulate unbounded soil for rigid embedded foundations undergoing vertical, horizontal, rocking and torsional motions. Parameters used in the new model are more efficiently determined without lengthy optimization analysis. Moreover, an optimal simplified model is selected through minimizing an error function which is clearly addressed and easily evaluated more than that used in most of conventional methods. The dynamic responses of the foundation-soil system using the new simplified model are compared with those obtained by the half-space theory and by the existing lumped-parameter models to examine the accuracy of the new model. Moreover, a time-domain validation for the proposed model applied to a building-soil interaction system subjected to vertical and horizontal excitations is also provided.

\section{THEORY OF EQUiVALENT MODELS}

This paper mainly investigates the dynamic response of a rigid embedded foundation undergoing vertical, horizontal, rocking and torsional motions. A series of equivalent models shown in Fig. 1 is used to simulate the unbounded soil. For vertical and horizontal motions, a single degree-of-freedom (SDOF) model is used. For rocking and torsional motions, a 2-DOF model is considered. The arrangement of the equivalent model includes a lumped mass (or inertia), a spring, and a dashpot in each directions; and two vertical eccentricities to consider coupling effects. For a foundation-soil system excited by harmonic forces with different frequencies, a group of equivalent models are developed by considering three criteria. The first criterion is equivalent static response, which establishes an equivalence of static displacement between the equivalent model and the foundation-soil system subject to a static force. The second 
criterion is equivalent magnification factor, which establishes an equivalence of dynamic magnification factor (i.e., ratio of displacement amplitude to static response) between the equivalent model and the foundation-soil system subject to a harmonic force. The last one is equivalent dissipated energy, which establishes an equivalence of dynamic dissipated energy between the equivalent model and the foundation-soil system subject to the harmonic force. Following the theory of equivalent models, the modeling parameters can be derived for each direction of motions. These parameters are summarized in the following.
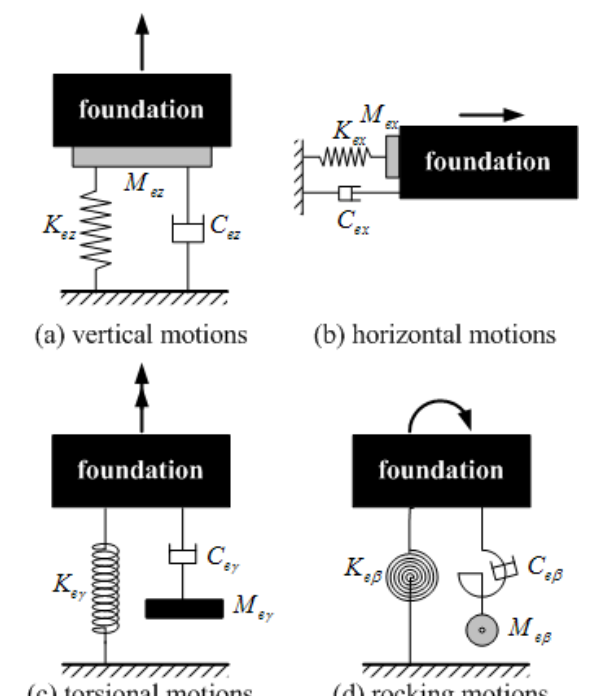

(b) horizontal motions

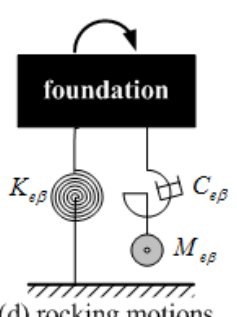

Fig. 1. A series of equivalent models.

For vertical motions, the modeling parameters with stiffness $K_{e z}$, damping coefficient $C_{e z}$ and lumped mass $M_{e z}$ can be shown as below.

$$
K_{e z}=K_{s z} ; C_{e z}=k_{e z} c_{z} \rho V_{s} R^{2} ; M_{e z}=\frac{k_{e z}\left(1-k_{z}\right)}{a_{0}^{2}} \rho R^{3}
$$

where $K_{s z}=$ static foundation stiffness of vertical motions; $V_{s}$ $=$ shear velocity of soil; $R=$ characteristic length of the foundation; $\rho=$ soil density; $k_{e z}=K_{e z} /(G R)=$ normalized static stiffness with a shear modulus $G$ of soil; $k_{z}$ and $c_{z}=$ dimensionless stiffness and damping coefficients of vertical impedances, respectively; and $a_{0}=\omega R / V_{s}=$ dimensionless frequency with a forcing frequency $\omega$.

For horizontal motions, the modeling parameters with stiffness $K_{e x}$, damping coefficient $C_{e x}$ and lumped mass $M_{e x}$ can be similarly expressed as below.

$$
K_{e x}=K_{s x} ; C_{e x}=k_{e x} c_{x} \rho V_{s} R^{2} ; M_{e x}=\frac{k_{e x}\left(1-k_{x}\right)}{a_{0}^{2}} \rho R^{3}
$$

where $K_{s x}=$ static foundation stiffness of horizontal motions; $k_{e x}=K_{e x} /(G R)=$ normalized static stiffness; $k_{x}$ and $c_{x}=$ dimensionless stiffness and damping coefficients of horizontal impedances, respectively.

For torsional motions, the modeling parameters with stiffness $K_{e \gamma}$ damping coefficient $C_{e \gamma}$ and lumped inertia $M_{e \gamma}$ can be written as below.

$$
\begin{aligned}
& K_{e \gamma}=K_{s \gamma} \\
& C_{e \gamma}=\frac{k_{e \gamma}\left[a_{0}^{2} c_{\gamma}^{2}+\left(1-k_{\gamma}\right)^{2}\right]}{a_{0}^{2} c_{\gamma}} \rho V_{s} R^{4} \\
& M_{e \gamma}=\frac{k_{e \gamma}\left[a_{0}^{2} c_{\gamma}^{2}+\left(1-k_{\gamma}\right)^{2}\right]}{a_{0}^{2}\left(1-k_{\gamma}\right)} \rho R^{5}
\end{aligned}
$$

where $K_{s \gamma}=$ static foundation stiffness of torsional motions; $k_{e \gamma}=K_{e \gamma} /\left(G R^{2}\right)=$ normalized static stiffness; $k_{\gamma}$ and $c_{\gamma}=$ dimensionless stiffness and damping coefficients of torsional impedances, respectively.

For rocking motions, the modeling parameters with stiffness $K_{e \beta}$, damping coefficient $C_{e \beta}$ and lumped inertia $M_{e \beta}$ can be similarly derived as follows.

$$
\begin{aligned}
& K_{e \beta}=K_{s \beta} \\
& C_{e \beta}=\frac{k_{e \beta}\left[a_{0}^{2} c_{\beta}^{2}+\left(1-k_{\beta}\right)^{2}\right]}{a_{0}^{2} c_{\beta}} \rho V_{s} R^{4} \\
& M_{e \beta}=\frac{k_{e \beta}\left[a_{0}^{2} c_{\beta}^{2}+\left(1-k_{\beta}\right)^{2}\right]}{a_{0}^{2}\left(1-k_{\beta}\right)} \rho R^{5}
\end{aligned}
$$

where $K_{s \beta}=$ static foundation stiffness of rocking motions; $k_{e \beta}=K_{e \beta}\left(G R^{2}\right)=$ normalized static stiffness; $k_{\beta}$ and $c_{\beta}=$ dimensionless stiffness and damping coefficients of rocking impedances, respectively. For coupled horizontal and rocking motions, the modeling parameters shown in (2) and (4) are to be further modified by considering the coupling impedances in the dynamic equations of motions. More details are illustrated in the previous work [12].

Note that for a foundation-soil system excited by harmonic forces corresponding to $N$ discrete frequencies, using the proposed method will result in $N$ equivalent models. An optimal model is then determined from the established equivalent models to obtain the most accurate frequency-magnification response for the foundation-soil system subjected to harmonic forces; i.e. the optimal model has the minimum error of dynamic magnification factors. An error function used is defined as

$$
\varepsilon_{o}=\sqrt{\sum_{j=1}^{N}\left(\frac{M_{o}-\bar{M}_{o}}{M_{o}}\right)_{j}^{2} \times p_{j}}
$$

where $M_{o}=$ dynamic magnification factor of the foundation-soil system; $\bar{M}_{o}=$ dynamic magnification factor of the foundation with an equivalent model; the weighting $p_{j}$ $=\left(M_{o}\right)_{j}$ corresponding to the $j$-th frequency point; $N=$ number of frequency points and the subscript $o$ denotes the direction of motion. This paper mainly investigates the dynamic response of a foundation-soil system using the optimal equivalent model to effectively simulate the unbounded soil.

\section{VALidATIONS ON Foundation-SOIL Systems}

Applications of the optimal equivalent model are to be investigated on the problem of foundation vibration in this 
section. Consider a rigid square foundation embedded in a slightly damped half-space with damping ratios $\zeta_{p}=0.0005$ and $\zeta_{s}=0.001$. The embedment depth of foundation is assumed to be 1.5 times of characteristic length $R$ (i.e., one half-side length) while the foundation mass is $5 \rho R^{3}$. Suppose that the rigid foundation is harmonically subjected to a vertical force, horizontal force, rocking moment, and torsion, respectively, with varied frequencies. A series of vertical, horizontal, rocking, and torsional impedance function presented by Mita and Luco [13] is used in the proposed method to develop the optimal equivalent model to simulate the damped half-space. The dynamic magnification factors for a foundation-soil system using the optimal equivalent model are to be verified with those obtained by the half-space theory. The dynamic magnification factor is defined as the ratio of dynamic displacement amplitude to static response when the foundation subjected to an external harmonic force or moment. In addition, similar comparisons are also made for existing lumped parameter models. The analyzed results are displayed in Fig. 2. For vertical, horizontal, rocking, and torsional motions, observe in Fig. 2 that the frequency-magnification responses obtained from the optimal equivalent model consist well with the half-space solutions even as the dimensionless frequency increases. For vertical motions, the magnification factors analyzed by the optimal equivalent model consist very well with those obtained by Barros and Luco's model (2 DOFs and 5 parameters), especially for the peak responses. For torsional motions, the results analyzed by the Wu and Chen's model (3 DOFs and 9 parameters) underestimate the torsional magnification factors in low-frequency range but accurately estimate the high-frequency responses. By contrast, the torsional responses obtained by the optimal equivalent model consist well with the half-space solutions in all considered frequencies. For horizontal motions, Wu and Chen's model (1 DOF and 3 parameters) overestimates the horizontal magnification factors in comparison with the half-space solutions. For rocking motions, Wu and Chen's model (3 DOFs and 9 parameters) underestimates the rocking magnification factors especially for responses around the resonance frequency. This feature can be markedly observed for the rigid foundation with a deep embedment ratio or a large mass ratio. By contrast, good agreements are observed again between the results obtained by the optimal equivalent model and the half-space solutions. Hence, the optimal equivalent model may be more accurate than Wu and Chen's model due to the coupling effects on horizontal and rocking motions are well considered in the proposed method. The presented results also reveal that the rotational responses of embedded foundations subjected to a harmonic moment may be significantly underestimated without considering the coupled horizontal and rocking motions. The optimal equivalent model developed may also effectively simulate the uniform half-space for the rigid embedded square foundation undergoing forced vibrations.

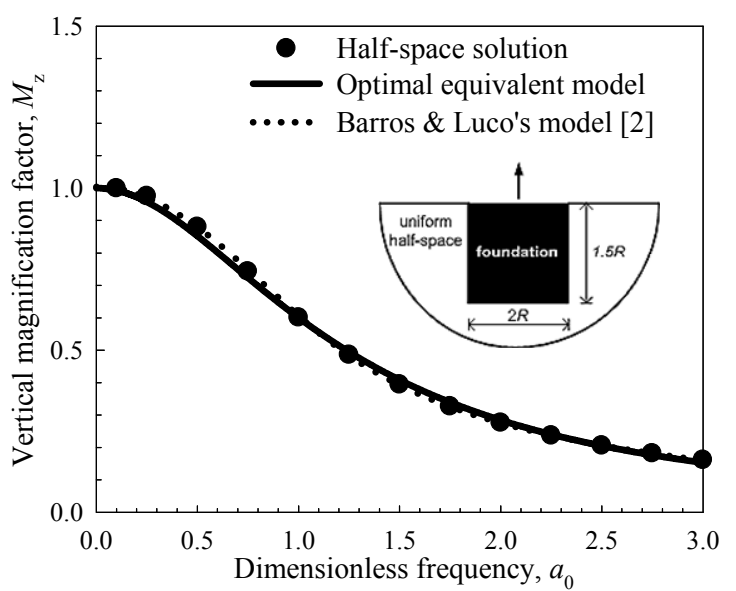

(a) vertical motions

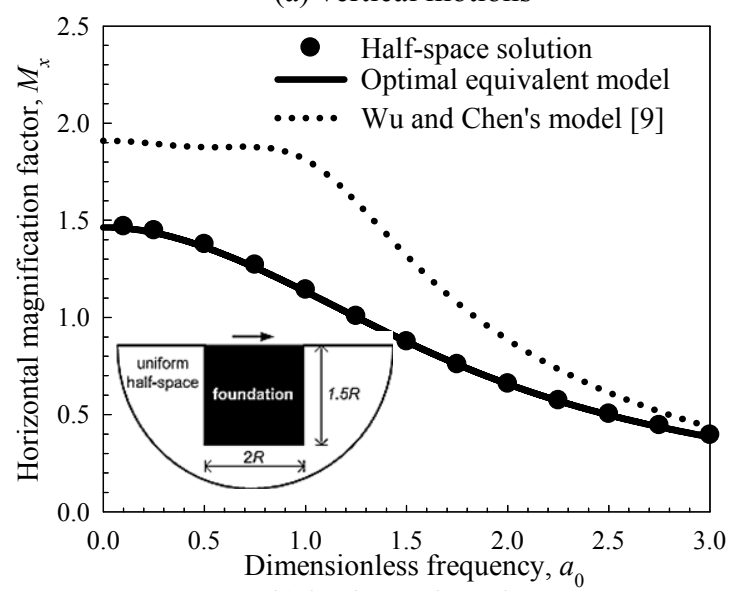

(b) horizontal motions

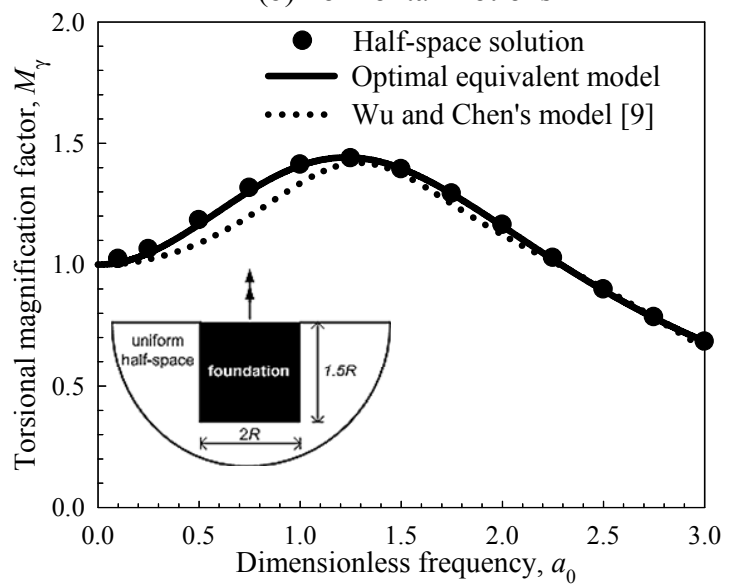

(c) torsional motions

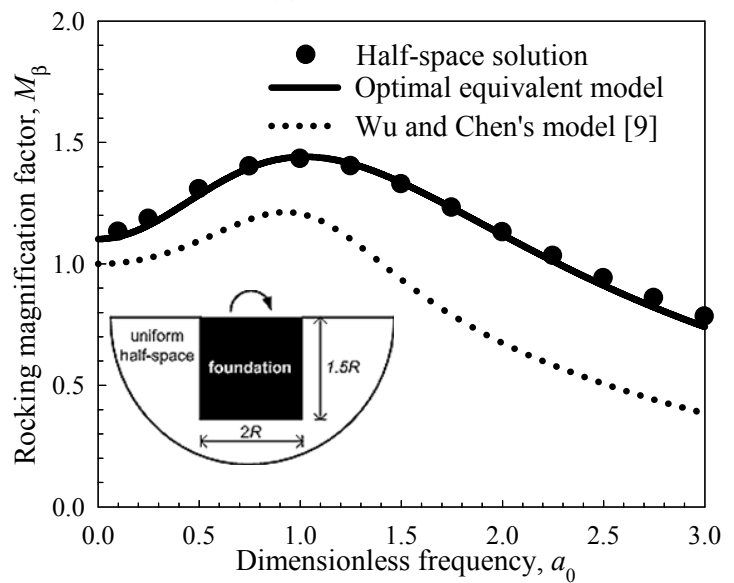

(d) rocking motions

Fig. 2. Dynamic response of foundation-soil systems. 


\section{VALidATIONS ON BUILDING-SoIL SySTEMS}

Applications of the optimal equivalent model are to be investigated on the problem of seismic excitation in this section. Considered an idealized 3-story building (shown in Fig. 3) embedded in a damped half-space with uniform story stiffness and mass while the associated system parameters are listed in Table I. In the analytical models, the horizontal ground acceleration history recorded from Station TCU045 during 1999 Taiwan Chi-Chi earthquake is to be considered. As shown in Fig. 4, the responses of a simplified building-soil system using the optimal equivalent model are to be obtained by a modal analysis method in the time domain, which are accomplished by a commercial program, SAP2000 [14]. Moreover, the responses of the real building-soil system are further analyzed by using a numerical program, SASSI [15]. This program SASSI features in using a special transmitting boundary to simulate the soil layer and a flexible volume method to solve dynamic soil-structure interaction problems. For the 3-storey structures, the horizontal acceleration history at the top floor is displayed in Fig. 5 while the associated floor response spectrum is shown in Fig. 6. Observe that the history responses of the simplified system mostly agree well with that of the real system. No marked differences on the accelerations or phases can be found. The spectral curves of the simplified system also consist well with that of the real system. In summary, for the building structure undergoing horizontal excitations, the seismic response of the simplified system agrees well with that of the real system.

\begin{tabular}{cl}
\multicolumn{1}{c}{ TABLE I: SYSTEM PARAMETERS OF NUMERICAL EXAMPLES } \\
\hline \hline Zone & \multicolumn{1}{c}{ Parameter } \\
\hline & Poisson's ratio: 0.25 \\
Uniform & Shear-wave velocity: $500 \mathrm{~m} / \mathrm{s}$ \\
Half-space & Material density: $2 \mathrm{Mg} / \mathrm{m}^{3}$ \\
& Damping ratio for $P$-wave: 0.0005 \\
& Damping ratio for $S$-wave: 0.001 \\
\hline & Side length: $20 \mathrm{~m}$ \\
& Embedment depth: $5 \mathrm{~m}$ \\
Square & Foundation mass: $5000 \mathrm{Mg}$ \\
& Foundation inertia: $177083 \mathrm{Mg}-\mathrm{m}^{2}$ \\
& Static stiffness: $4.16 \times 10^{7} \mathrm{kN} / \mathrm{m}$ \\
& Static stiffness: $5.58 \times 10^{9} \mathrm{kN}-\mathrm{m}$ \\
\hline Upper structure & Storey mass: $2000 \mathrm{Mg}$ \\
& Storey inertia: $66708 \mathrm{Mg}-\mathrm{m}^{2}$ \\
& Storey stiffness: $2.08 \times 10^{7} \mathrm{kN} / \mathrm{m}$ \\
& Model damping ratio: $5 \%$ \\
\hline \hline
\end{tabular}

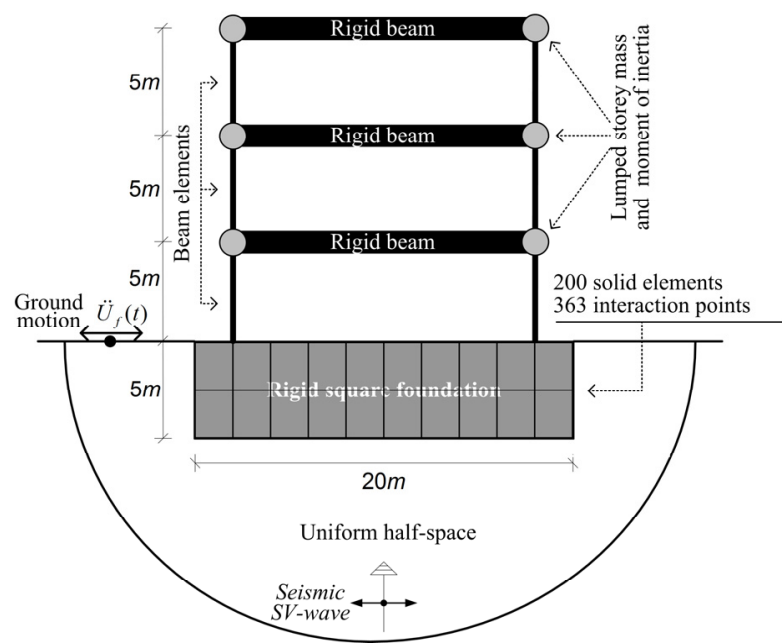

Fig. 3. Case study of an idealized three-story building: Real System

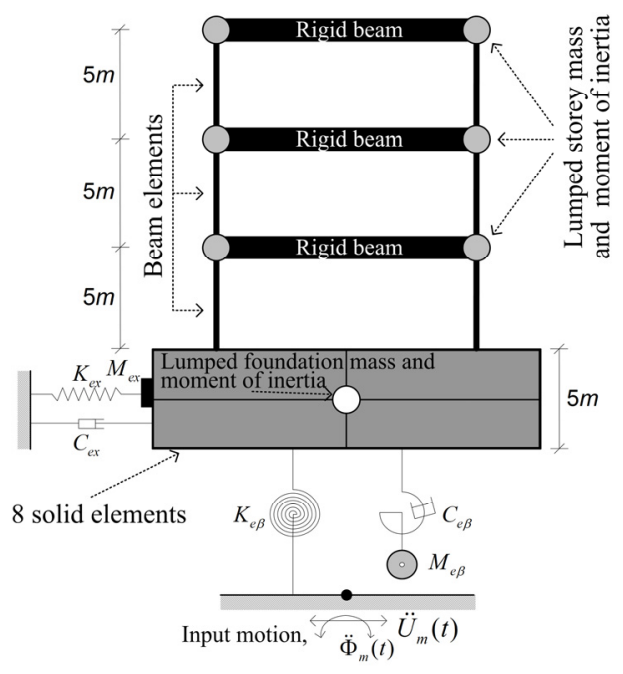

Fig. 4. Case study of an idealized three-story building: Simplified System

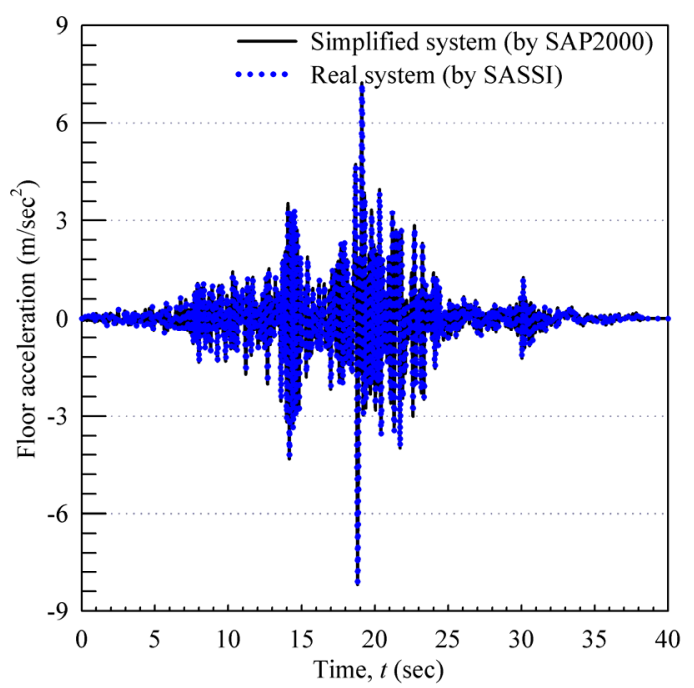

Fig. 5. Horizontal acceleration history of the top floor.

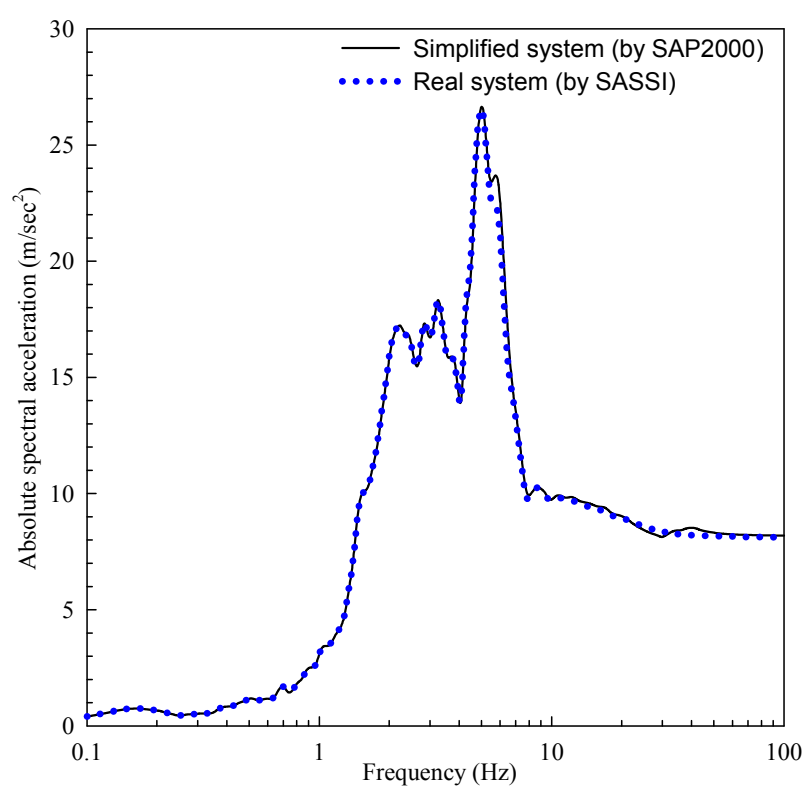

Fig. 6. Horizontal response spectrum of the top floor.

\section{Conclusions}

A simplified model is presented to simulate the unbounded soil for rigid embedded foundations. The modeling parameters are determined by the theory of equivalent model 
without using lengthy optimization. For a foundation-soil system subjected to forced vibrations, the dynamic magnification responses analyzed by using the optimal equivalent model consist well with those obtained from the half-space theory and existing lumped-parameter models. For an idealized building structures subjected to horizontal excitations, the floor response of a simplified building-soil system using the proposed model may also agree with those obtained from the numerical program. This new method may be effectively applied to practical problems involving soil-structure interaction, such as machine foundation vibration and seismic structural analysis.

\section{REFERENCES}

[1] J. P. Wolf and D. R. Somaini, "Approximate dynamic model of embedded foundation in time domain," Earthquake Engineering \& Structural Dynamics, vol. 14, no. 5, pp.683-703, Sep. 1986.

[2] F. C. P. de Barros and J. E. Luco, "Discrete models for vertical vibrations of surface and embedded foundations," Earthquake Engineering \& Structural Dynamics, vol. 19, no. 2, pp. 289-303, Feb. 1990.

[3] W. Y. Jean, T. W. Lin, and J. Penzien, "System parameters of soil foundation for time domain dynamic analysis," Earthquake Engineering \& Structural Dynamics, vol. 19, no. 4, pp. 541-553, May 1990.

[4] J. P. Wolf, "Consistent lumped-parameter models for unbounded soil: physical representation," Earthquake Engineering \& Structural Dynamics, vol. 20, no. 1, pp. 11-32, Jan. 1991.

[5] J. P. Wolf, "Consistent lumped-parameter models for unbounded soil: Frequency-independent stiffness, damping and mass matrices," Earthquake Engineering \& Structural Dynamics, vol. 20, no. 1, pp. 33-41, Jan. 1991.

[6] J. P. Wolf and A. Paronesso, "Lumped-parameter model for a rigid cylindrical foundation embedded in a soil layer on rigid rock," Earthquake Engineering \& Structural Dynamics, vol. 21, no.12, pp. 1021-1038, Dec. 1992

[7] J. P. Wolf, Foundation Vibration Analysis Using Simple Physical Models, Englewood Cliffs, New Jersey: Prentice-Hall, 1994, ch. 2.

[8] J. P. Wolf, "Spring-dashpot-mass models for foundation vibrations," Earthquake Engineering \& Structural Dynamics, vol. 26, no. 9, pp. 931-949, Sep. 1997.

[9] W. H. Wu and C. Y. Chen, "Simple lumped-parameter models of foundation using mass-spring-dashpot oscillators," Journal of Chinese Institute of Engineers, vol. 24, no. 6, pp. 681-697, Nov. 2001.

[10] W. H. Wu and W. H. Lee, "Systematic lumped-parameter models for foundations based on polynomial-fraction approximation," Earthquake Engineering \& Structural Dynamics, vol. 31, no. 7, pp. 1383-1412, July 2002.
[11] W. H. Wu and W. H. Lee, "Nested lumped-parameter models for foundation vibrations," Earthquake Engineering \& Structural Dynamics, vol. 33, no. 9, pp. 1051-1058, July 2004.

[12] J. Y. Shi, "Simplified Models for Dynamic Soil-Structure Interaction Systems," Ph. D Dissertation, Dept. Construction Eng., National Taiwan University of Science and Technology, Taiwan, 2006.

[13] A. Mita and J. E. Luco, "Impedance functions and input motions for embedded square foundations," Journal of Geotechnical Engineering, ASCE, vol. 115, no.4, pp.491-503, April 1989.

[14] E. L. Wilson and A. Habibullah, "SAP2000 Nonlinear V.8 - Integrated Software for Structural Analysis and Design (CD-ROM)," Computer and Structures, Inc., Berkeley, Calif., 2002.

[15] J. Lysmer, M. Tabatabaie-Raissi, F. Tajirian, S. Vahdani, and F. Ostadan, "SASSI: a system for analysis of soil-structure interaction problems," Report UCB/GT81-02, University of California, Berkeley, 1981.

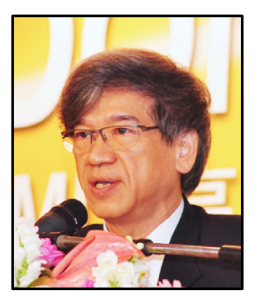

Shi-Shuenn Chen received his doctorate in civil engineering from the University of California, Berkeley, is former President of the National Taiwan University of Science and Technology (Taiwan Tech). He established the Taiwan Building Technology Center in Taiwan while pursuing a First-Class University to foster the development of building technologies at the cutting edge of research. Dr. Chen serves currently as the president of the Chinese Institute of Civil and Hydraulic Engineering, the president of the Association of National Universities of Science and Technology, as well as the president of the Chinese Association for Cross-Strait Exchanges and Cooperation in Higher and Vocational Education. $\mathrm{He}$ is also the editor-in-chief of the Journal of the Chinese Institute of Engineers. Dr. Chen also served previously as a member of the Public Construction Commission, under the Executive Yuan, and of the Supervisory Board of Science Education, under the Ministry of Education of the ROC. Dr. Chen's previous positions also include those of deputy director general in the National Expressway Engineering Bureau, president of the Chinese Association of Structure Engineers. Honors received by Dr. Chen include the Outstanding Engineering Professor Award from the Chinese Institute of Engineers and the Excellent Research Paper Award from the Chinese Institute of Civil and Hydraulic Engineering.

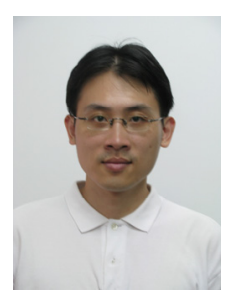

Jun-Yang Shi received his doctorate in construction engineering from the National Taiwan University of Science and Technology (Taiwan Tech). Currently, he is a post-doctoral researcher in Taiwan Building Technology Center in Taiwan Tech. Dr. Shi has studied the following areas: earthquake engineering and structural dynamics. 